\title{
Solução simultânea de problemas logísticos de localização de depósitos e centralização de estoques
}

Ricardo Ferrari Pacheco

LuCIANo ZorzetT CiRqueira

Universidade Católica de Goiás

\begin{abstract}
Resumo
A elaboração de uma estratégia eficiente de distribuição envolve os passos de determinação do número de depósitos e sua localização, a estratégia de alocação de estoques dos produtos em cada um dos depósitos e a elaboração de roteiros de distribuição dos produtos dos depósitos aos pontos finais de venda.

Devido à complexidade envolvida em cada um desses passos, essas decisões têm sido tomadas de forma hierárquica e seqüencial. Esta abordagem leva a soluções subótimas, pois o problema é dividido em subproblemas e a solução de um sub-problema afeta os demais. Este trabalho tem como objetivo propor um modelo integrado para a solução simultânea dos problemas de localização de depósitos e alocação de estoques e propõe um algoritmo heurístico baseado em abordagem evolucionária para solução do mesmo. 0 modelo proposto foi aplicado em um problema real envolvendo o replanejamento logístico de uma rede de farmácias, demonstrando sua viabilidade técnica.
\end{abstract}

Palavras-chave

Logística, localização de depósitos, centralização de estoques, modelagem.

\section{Simultaneous solution of logistic problems envolving warehouse location and centralization of inventories}

\begin{abstract}
The elaboration of an efficient distribution strategy involves steps like determination of warehouse number and its location, the strategy of inventory products allocation in each warehouse and elaboration of routes for products distribution, from warehouses to points of sale.

Due the complexity involving each one these steps, decisions have been done in a hierarchical and sequential way. This traditional approach conduces to sub-optimal solutions, since the problem is divided in sub-problems and each sub-problem solution affect the others. This paper proposes a single model for solve the warehouse location and centralization of inventories problems in a simultaneous mode and proposes a heuristic algorithm for the problem based on evolutionary approach. Proposed model was applied on a real problem involving the re-planning of a drugstore network, showing its technical viability.
\end{abstract}

Key words

Logistics, warehouse location, centralization of inventories, modeling. 


\section{INTRODUC̣̃̃O}

Atualmente, as empresas vêm cada vez mais buscando alternativas que propiciem a redução de seus custos operacionais, a melhoria de seu desempenho e, conseqüentemente, do nível de serviço oferecido ao cliente. Bowersox et al. (2006) afirmam que fornecedores e distribuidores de produtos e serviços em todo o mundo reconhecem a necessidade de estruturar suas estratégias de distribuição buscando a melhoria da eficiência em transporte e do nível de serviço ao cliente.

Freqüentemente, o processo de estruturação da malha logística é subdividido em problemas bastante definidos e estanques como: problemas de localização de instalações, problemas de alocação de estoques aos depósitos e problemas de roteirização de veículos. Tais problemas são intimamente relacionados, mas não são tratados de forma integrada pelos modelos atualmente utilizados, devido, principalmente, à dificuldade computacional envolvida. Este trabalho propõe um algoritmo que aborda simultaneamente os problemas de localização de instalações e de centralização ou descentralização de estoques e demonstra as vantagens dessa abordagem.

A estrutura do trabalho é a que segue: inicialmente é apresentada a estrutura metodológica utilizada no trabalho; a seguir discutem-se os problemas de localização de instalações, centralização e descentralização de estoques, de roteirização de veículos e abordagens conjuntas; continuando, é apresentado o modelo proposto e mostrada a implementação computacional do modelo. Na seqüência, apresenta-se um problema real no qual o modelo foi aplicado e demonstramse os resultados obtidos, finalmente são tecidas algumas conclusões.

\section{A ESTRUTURA METODOLÓGICA DO TRABALHO}

O trabalho aqui apresentado tem duas questões de pesquisa principais. A primeira diz respeito à viabilidade técnica de se modelar conjuntamente os problemas de localização de instalações e centralização ou descentralização de estoques em uma malha logística. A segunda questão diz respeito ao ganho que se pode esperar com a aplicação do modelo proposto.

Este trabalho pode ser classificado do ponto de vista de sua natureza como uma pesquisa aplicada, visto que "objetiva gerar conhecimentos para aplicação prática e dirigida à solução de problemas específicos" (SIVA e MENEZES, 2005). Em relação aos seus objetivos, é exploratório, pois visa, ainda de uma forma preliminar, analisar a viabilidade técnica e os ganhos que se podem esperar com a utilização do modelo proposto. Com relação à abordagem, o trabalho se caracteriza como pesquisa quantitativa. Já os procedimentos técnicos utilizados no trabalho abrangem pesquisa bibliográfica, modelagem matemática e estudo de caso.
É importante salientar que a abordagem baseada em estudo de caso não tem como objetivo a generalização estatística dos resultados e sim investigar com profundidade fenômenos e seu contexto (BRYMAN, 1989). Assim, o objetivo da aplicação da metodologia de estudo de caso neste trabalho foi o de verificar se o modelo aqui proposto era realmente exeqüível em um caso real, e quais os resultados que se poderiam obter com a utilização do modelo proposto.

O trabalho aqui descrito foi dividido em cinco etapas:

A primeira etapa compreendeu uma revisão bibliográfica acerca de modelos matemáticos para localização de instalações e gestão e compartilhamento de estoques, bem como possíveis modelos conjuntos existentes.

A segunda etapa do trabalho compreendeu a elaboração do modelo matemático utilizado para localizar os centros de distribuição e os estoques.

A terceira etapa do trabalho compreendeu a implementação do modelo matemático elaborado na segunda etapa. Para tal, foi desenvolvido um aplicativo heurístico baseado em algoritmos genéticos em VBA (Visual Basic for Applications). A terceira etapa abrangeu ainda a validação computacional do modelo desenvolvido.

A quarta etapa do trabalho compreendeu a coleta dos dados necessários à execução do modelo em um caso real estudado. Para tal, efetuaram-se coletas nas bases de dados da rede logística estudada, entrevistas com diretores e coletas a campo nos pontos candidatos à localização.

A quinta e última etapa do trabalho tratou da parametrização e execução do modelo, das análises dos resultados e da elaboração das conclusões obtidas com a utilização do modelo no caso estudado.

\section{REVISÃO BIBLIOGRÁFICA}

Neste tópico é feita uma breve revisão sobre a modelagem de problemas de localização de instalações, centralização e descentralização de estoques e modelos logísticos conjuntos.

\section{Localização de Instalações}

Os problemas da localização de instalações vêm sendo estudados há bastante tempo no âmbito de serviços (FITZSIMMONS \& FITZSIMMONS, 2000), no âmbito da logística (BOWERSOX \& CLOSS, 2001; SIMCHI-LEVI et al, 2000; BALLOU, 1993), e no âmbito da pesquisa operacional (GOLDBARG, 1987). A definição da localização de uma facilidade, tal como uma fábrica, ou de um centro de distribuição é uma tarefa geralmente delegada aos encarregados de logística (BOWERSOX \& CLOSS, 2001). Trata-se, na maioria dos casos, de escolher, entre um número finito de alternativas possíveis, aquela que demons- 
tre ser a mais vantajosa no atendimento dos objetivos que norteiam a implantação da empresa (VALE, 1975). Além do tradicional papel de criar barreiras à entrada de competidores e de gerar demanda, a localização também afeta as dimensões estratégicas de flexibilidade, posicionamento competitivo e gerenciamento da demanda. Segundo Bowersox \& Closs (2001), ganhos com economia de escala na produção e redução no custo de transporte são objetos de atenção nos estudos de localização de indústrias. Nos últimos anos, os estudos de localização têm abrangido também projetos de canais logísticos, como resultado da globalização de fontes de suprimentos e de considerações de marketing (GOUVEIA, 1995). Operações globais aumentaram a complexidade das decisões relacionadas com os canais logísticos, com a definição de alternativas e com os custos logísticos respectivos. O fenômeno da globalização aumentou substancialmente a importância do estudo de localização. A flexibilidade de localização de uma instalação de serviço é ainda uma medida do grau de reação do serviço à mudança no ambiente econômico e envolve aspectos de capital intensivo com comprometimentos em longo prazo. É essencial selecionar locais que possam responder a futuras mudanças econômicas, demográficas, culturais e competitivas. Por exemplo, alocando instalações em vários Estados, é possível reduzir o risco total decorrente de crises financeiras desencadeadas por problemas regionais. Essa abordagem de portfolio para múltiplas localizações pode ser reforçada pela definição de locais individuais com demandas quase inelásticas.

Para Jenkins (1968), a localização de indústrias envolve o uso de muito julgamento, arte e intuição. Os analistas da decisão devem seguir uma lista de verificação dos fatores que podem auxiliar a diminuir a amplitude de suas escolhas. Alguns fatores são: leis de zoneamento locais, a atitude da comunidade e do governo local com relação à facilidade; custo para desenvolver e conformar o terreno; custo da construção; disponibilidade; salários; ambiente e produtividade da mão-de-obra local; taxas relativas ao local e à operação da facilidade; segurança do local (furto, fogo, inundação, etc.); valor promocional do local; taxas de seguro; disponibilidade de financiamento e congestionamento de tráfego nas redondezas do local. Alguns autores denominam macrolocalização a determinação da região propícia à instalação e microlocalização a determinação específica da área a ser adquirida. Este trabalho aborda a macrolocalização.

\section{Classificações dos Problemas de Localização}

Existem inúmeras abordagens e aplicações para o problema de localização. Os problemas vão desde a definição de uma localização única no plano contínuo, até a definição de múltiplas localizações em um grafo, de modo a atender determinadas restrições.

O problema de centro de gravidade se caracteriza por tratar da localização de uma única facilidade no plano contínuo. O objetivo é encontrar a melhor localização que minimize os custos de transporte, dados pelo somatório das distâncias multiplicadas pelos fluxos a movimentar e pelos custos de movimentação (BOWERSOX \& CLOSS, 2001). Pode-se utilizar a métrica metropolitana, retangular ou euclidiana. A segunda forma torna o problema não-linear (pois a distância é quadrática) e foi denominado problema de centro de gravidade (COG) ou problema generalizado de Weber, em homenagem a Alfred Weber, que primeiro o formulou em 1909.

\section{- reqüentemente, o processo de estruturação da malha logística é subdividido em problemas bastante definidos e estanques como: problemas de localização de instalações, problemas de alocação de estoques aos depósitos e problemas de roteirização de veículos.}

Os problemas de localização utilizando teoria de grafos apresentam inúmeras aplicações reais. O problema de particionamento de grafos é um caso particular do problema de recobrimento e possui diversas aplicações em problemas de localização. Daskin \& Stern (1981) aplicaram o modelo de cobertura na solução do problema da localização de serviços médicos, e Goldbarg (1987), na localização de radares.

Já o problema de cobertura no plano contínuo se caracteriza por ser mais complexo, pois existe um número infinito de pontos candidatos (WHITE \& CASE, 1980). Dado um conjunto de pontos a serem atendidos pela facilidade, a distância que o cliente mais distante deveria percorrer para alcançar a instalação é denominada distância máxima de serviço. Os pontos cujas distâncias são iguais ou inferiores à distância máxima de serviço encontram-se dentro da área de cobertura da facilidade. O problema que tem como objetivo encontrar o número mínimo e a localização das instalações que irão servir todos os pontos da demanda, abrangendo-os na área de cobertura de algumas facilidades, é conhecido como problema de recobrimento mínimo. Benvenistes (1982) propõe um modelo no qual a área de cobertura é retangular. Já o problema de localização de máxima cobertura se caracteriza 
por determinar, dado um número $p$ máximo de facilidades a localizar e valores de demanda $a_{i}$, localizados em cada vértice $i$, quais as localizações das facilidades de modo a maximizar a demanda atendida (CHURCH \& REVELLE, 1974).

O problema que busca determinar, dentre as $n$ possíveis, as $\mathrm{k}(\mathrm{k}<=\mathrm{n})$ localizações, tal que a distância mínima entre as mesmas seja máxima, é denominado problema de k-dispersão discreta. Os exemplos de aplicação são geralmente relacionados aos problemas de localização de facilidades cuja proximidade é indesejável. Church \& Garfunkel (1978) aplicaram o modelo em problemas de localização de depósitos de lixo e Berman et al. (1996), em problemas de localização de prisões e instalações militares.

No problema de k-servos, a facilidade percorre uma trajetória em um grafo, atendendo cada um dos nós. Ele se distingue do problema de roteirização pelo fato de o ponto final ser distinto do inicial e de a solução depender da decisão do ponto inicial do trajeto. Church \& Eaton (1987) utilizaram um modelo de k-servos para determinar a localização de clínicas e hospitais.

\section{Problema de Centralização ou Descentralização de Estoques}

Slack et al. (1999) definem estoque como sendo a acumulação de recursos materiais em um sistema de transformação, sendo que estes algumas vezes podem ser utilizados para descrever qualquer recurso armazenado. Moreira (1996) define estoque como quaisquer quantidades de bens físicos que sejam conservados, de forma improdutiva, por algum intervalo de tempo.

Slack et al. (1999) relatam que o desequilíbrio entre o fornecimento e a demanda gera quatro tipos diferentes de estoque: isolador ou de segurança, de ciclo, de antecipação e de canal. De acordo com Motta (1990), a função do estoque com relação à gestão da produção é abastecer o fluxo produção-venda de forma continua e uniforme, sem que haja interrupções. Para Ballou (1993), o estoque tem as finalidades de melhorar o nível de serviço, incentivar economias na produção, permitir economias de escala nas compras e no transporte, agir como proteção contra aumento de preços, proteger a empresa de incertezas na demanda e no tempo de resuprimento e servir como segurança contra contingências.

Existem boas razões tanto para distribuir o estoque em diversos centros de distribuição próximos do consumidor, quanto para alocá-lo em um número menor de centros, cada um deles com uma área geográfica de cobertura maior, o que faz com que esta decisão deva ser criteriosamente analisada.

Segundo Christopher (1999), com a consolidação dos estoques em menos locais, podem-se reduzir substancialmente as necessidades totais dos mesmos, além de se reduzir custos fixos de manutenção de depósitos. Smykay (1973) observa que o estoque de segurança armazenado em um único ponto centralizado é aproximadamente a razão do estoque total de segurança dos vários locais possíveis pela raiz quadrada do número total de localizações. Maister (1976) formaliza a "lei da raiz quadrada" e estabelece que, em geral, a relação entre estoque centralizado e descentralizado é igual a: $\sqrt{ }_{m} / \sqrt{ }_{n}$, sendo $m$ o número de localizações após a consolidação e $n$ o número de localizações anteriores à consolidação. Segundo o autor, essa lei pode ser aplicável tanto ao cálculo da redução do estoque de segurança, quanto ao cálculo de estoques de manutenção, quando for empregado o lote econômico para pedido de ressuprimento.

Tallon (1993) também reconhece que a centralização reduz o nível dos estoques de segurança num ambiente de incerteza. Porém, observa que um maior grau de centralização pode aumentar as distâncias para os mercados e, conseqüentemente, prejudicar o atendimento ao cliente e provocar perda de mercado. Segundo o autor, as reduções no nível de serviço poderiam ser minimizadas por meio de investimentos visando maior agilidade no sistema de transportes ou no desempenho do processamento de pedidos, o que pode aumentar custos. As incertezas enfrentadas pela empresa poderiam também ser reduzidas por meio de acordos contratuais com clientes e fornecedores.

Para Wanke \& Figueredo (2000), são duas as características do produto que impactam na decisão de centralização ou descentralização dos estoques. Primeiramente, quanto maior for o valor agregado, maior será a propensão para centralização dos estoques numa única instalação, a fim de reduzir a duplicidade de custos associados à manutenção de estoques de segurança em diversas localidades. Em segundo lugar, quanto maior o grau de obsolescência, maior será a propensão para centralização dos estoques, a fim de reduzir os riscos de não venda. Segundo Simchi-Levi et al. (2000), como regra genérica, os produtos que possuem alto valor e baixa demanda devem, de modo geral, ser estocados em um depósito central. Já os itens de baixo custo que possuem alta demanda devem ser estocados de forma descentralizada. Ainda segundo o mesmo autor, quanto maior o coeficiente de variação do item, maior será o benefício obtido na sua centralização.

Zinn, Levy e Bowersox (apud SILVEIRA, 2002), aprofundaram as análises de Eppen (1979), passando a incluir a análise de demandas correlacionadas negativamente. Verificaram que estas geram ainda maiores reduções de estoque pela centralização do que as conseguidas com demandas não correlacionadas. Além disso, desenvolveram o modelo de portfolio effect $(\mathrm{PE})$, definido como "a redução percentual nos estoques de segurança conseguida através da centralização de estoques". Os autores demonstraram que a "lei da raiz quadrada" é um caso especial do portfolio effect. 
Ballou (1981) fez a comparação da "lei da raiz quadrada" com valores determinados por equações de regressão, obtidas através de dados reais de várias empresas, concluindo que esta lei é excessivamente otimista quanto à redução de estoques de segurança, na maioria dos casos. Mahmoud (1992) também desenvolveu um procedimento para cálculo do ganho pela centralização de estoques baseado no modelo do portfolio effect, visando selecionar um esquema de consolidação ótimo. Já Tallon (1993) estudou a influência da variabilidade do lead time no modelo do portfolio effect.

\section{Problemas Conjuntos}

Os problemas de localização, centralização ou descentralização de estoques e roteirização de veículos apresentam interfaces importantes, discutidas a seguir.

A definição, de forma isolada, da localização dos depósitos pode propiciar custos elevados de alocação dos estoques, pois a determinação do número de depósitos deveria considerar, e geralmente não o faz, as vantagens da centralização em termos de redução de estoques de segurança e redução dos custos fixos de instalações, contra os aumentos dos custos de distribuição e aumento dos leadtimes de entrega.

O número de depósitos pode ter um impacto significativo no desempenho dos roteiros de distribuição. Quanto maior o número de depósitos, geralmente mais eficientes são os roteiros de distribuição. Por outro lado, o aumento do número de depósitos leva ao aumento dos custos fixos de instalação. Já a escolha da localização dos depósitos, considerando-se apenas custos imobiliários e isenções fiscais, pode gerar roteiros de distribuição inadequados.

A centralização de estoques reduz os estoques de segurança, mas geralmente provoca aumento dos custos de distribuição. Esta decisão geralmente considera as economias de estoques e os custos de distribuição, levando em conta apenas as distâncias entre os depósitos e os clientes, sem considerar os possíveis roteiros de distribuição. A alocação de estoques aos depósitos existentes pode propiciar roteiros de distribuição inadequados, especialmente em casos de janelas de tempo ou veículos com capacidade limitada.

Assim, percebe-se que os problemas de localização, centralização de estoques e distribuição de mercadorias são intimamente relacionados, mas geralmente não são tratados de forma integrada, devido, principalmente, à dificuldade computacional envolvida. Mais recentemente, alguns autores têm proposto integrar dois problemas em um único modelo. O modelo conjunto mais comumente encontrado integra os problemas de localização e roteirização de veículos (location-routing problem ou LRP).

Sambola (2003) apresenta um interessante trabalho no qual são apresentadas, implementadas e analisadas modelagens heurísticas baseadas em busca tabu para o problema conjunto de localização de depósitos e roteirização de veículos (LRP), tanto no caso determinístico, quanto estocástico. Alguns algoritmos exatos têm sido propostos para o LRP no caso de um único depósito e veículos sem limitação de capacidade (AVERBAKH \& BERMAN, 1994) e com limitação de capacidade (CHIEN, 1993). Abordagens para o caso de múltiplos depósitos e veículos capacitados também foram propostas em Laporte et al. (1998). Não foram localizados trabalhos que tratem simultaneamente dos problemas de localização de depósitos e alocação de estoques, como faz o modelo aqui proposto.

\section{ssim, percebe-se que os problemas de distribuição de mercadorias são intimamente relacionados, mas geralmente não são tratados de forma integrada, devido, principalmente, à dificuldade computacional envolvida.}

\section{O MODELO PROPOSTO}

Nesta seção é apresentada a modelagem matemática desenvolvida. O modelo matemático baseia-se em localização discreta e combinação de alternativas de atendimento. O objetivo do modelo é a maximização da função na qual do total de vendas são subtraídos os custos fixos de instalação dos centros de distribuição e a diferença dos custos dos estoques de segurança nos casos descentralizado e centralizado. Os pontos-de-venda existentes podem operar de duas formas: mantendo seus próprios estoques descentralizados, ou por meio de centros de distribuição, nos quais os estoques dos pontos-de-vendas atendidos são centralizados. O modelo irá buscar criar centros de distribuição que maximizem a cobertura dos pontos-de-venda, desde que a economia obtida com a centralização dos estoques compense os custos fixos da instalação desses centros de distribuição. Para estimar o custo do estoque de segurança centralizado será utilizada a equação de Maister (1976). As principais características do modelo são:

- Cada centro de distribuição (CD) possui capacidade limitada e distinta.

- Cada CD instalado (ativo) possui um custo fixo. 
- O número de CDs possíveis está entre o número mínimo (Nmin) e o número máximo (Nmax).

- Os pontos de venda localizados dentro da área de cobertura de um $\mathrm{CD}$, definida pela distância de serviço $S$, poderão ter seu estoque centralizado em um dos CDs que o cobrem, caso haja capacidade disponível suficiente neste CD.

\section{A representação matemática do modelo}

Seja $\mathrm{S}$ a distância de serviço definida para que um pontode-venda seja coberto por um CD, $i=\{1, \ldots M\}$ o conjunto de áreas a serem atendidas, $j=\{1, \ldots N\}$ o conjunto de locais em potencial onde os CDs podem ser instalados, $L_{i}$ o lucro do ponto de venda $i$ e $c_{j}$ o custo fixo de instalação do CD na localização $j$. Seja $y_{i j}=1$ se o ponto $i \in M$ é atendido por um CD localizado em $j \in N$ considerando-se a distância de cobertura $S$, e $y_{i j}=0$ em caso contrário. Considere também que serão alocados $P \mathrm{CDs}$, sendo $P \geq \mathrm{Nmim}$ e $P \leq \mathrm{Nmax}$. Considere $x_{j}=1$ se o ponto j possui um CD ativo e $x_{j}=0 \mathrm{em}$ caso contrário. Considere $y_{i}=1$ se o ponto-de-venda $i$ for coberto por algum $\mathrm{CD}$ e $y_{i}=0$ caso contrário. Seja $e_{i}$ o custo do estoque de segurança no ponto-de-venda $i$ e $e_{j}$ o custo do estoque de segurança centralizado no $\mathrm{CD} j$. Seja $v_{i}$ o volume ocupado pelo estoque de segurança no ponto-de-venda $i$ e $C_{j}$ a capacidade de armazenagem do $\mathrm{CD} j$. A representação matemática do problema é dada por:

$\operatorname{Max}$

$$
Z=\left(\sum_{i=1}^{M} L_{i}\right)-\left(\sum_{j=1}^{N} c_{j} x_{j}\right)-\left(\sum_{i=1}^{M} e_{i}-\sum_{j=1}^{N} e_{j} x_{j}\right)
$$

Sujeito a:

$\begin{array}{ll}\sum_{j=1}^{N}\left(y_{i j} * x_{j}\right)-y_{i} \geq 0 & i=1 . . M \\ \sum_{j=1}^{N} y_{i j} \leq 1 & i=1 . . M \\ \sum_{j=1}^{N} x_{j}=P & j=1 . . N \\ N_{\min } \leq P \leq N_{\max } & j=1 . . N \\ e_{j}=\frac{\sum_{i=1}^{M}\left(e_{i} y_{i j}\right)}{\sqrt{\sum_{i=1}^{M} y_{i j}}} \\ \sum_{i=1}^{M} v_{i}\left(\begin{array}{l}\sum_{i=1}^{M}\left(e_{i} y_{i j}\right) \\ e_{j}\end{array} \leq C_{j} \quad j=1 . . N\right.\end{array}$

$\begin{array}{ll}\mathrm{x}_{\mathrm{j}} \in\{0,1\} & \mathrm{j}=1 . . \mathrm{N} \\ \mathrm{y}_{\mathrm{j}} \in\{0,1\} & \mathrm{i}=1 . . \mathrm{M} \\ \mathrm{y}_{\mathrm{ij}} \in\{0,1\} & \mathrm{i}=1 . . \mathrm{M}, \mathrm{j}=. . \mathrm{N}\end{array}$

A função objetivo do modelo matemático (4.1) é dada pela soma dos lucros dos pontos de venda (PV) subtraídos os custos fixos anuais $\left(c_{j}\right)$ de cada CD ativo no sistema e da diferença entre os custos de estocagem descentralizado e centralizado.

A restrição (4.2) expressa que se $y_{i j}=1$, ou seja, se o ponto $i$ é atendido pelo $\mathrm{CD}$ em $\mathrm{j}$, e caso $y_{i}=1$ (ou seja, o ponto foi atendido), logo existe algum $x_{j}=1$ (ou seja, algum CD deve estar ativo). Já a restrição (4.3) obriga que um pontode-venda seja atendido por no máximo um CD.

A restrição (4.4) determina que existirão $P$ pontos ocupados por CDs ativos. A restrição (4.5) determina a quantidade mínima e máxima de centros de distribuição que o sistema deve conter.

A restrição (4.6) determina que o estoque de segurança centralizado no CD $j$ é calculado pela somatória dos estoques existentes anteriormente nos pontos-de-venda atendidos pelo $\mathrm{CD}$ dividido pela raiz quadrada do número de pontos atendidos pelo $\mathrm{CD}$.

A restrição (4.7) expressa que o volume de estoque em cada $\mathrm{CD}$ não deve ser superior à sua capacidade. $\mathrm{O}$ volume do estoque no $\mathrm{CD}$ é inferior à soma dos volumes dos estoques descentralizados e é estimado pela mesma proporção da redução dos estoques de segurança causadas pela centralização.

As restrições (4.8), (4.9) e (4.10) expressam que as variáveis de decisão são binárias.

\section{A IMPLEMENTAC̣ÃO DO ALGORITMO}

Neste trabalho, a técnica de solução adotada foi heurística e baseada em algoritmos genéticos. O algoritmo foi implementado utilizando-se o ambiente VBA (Visual Basic for Applications).

\section{O algoritmo de solução}

O algoritmo tem início com a criação de uma população inicial de soluções viáveis. Cada indivíduo da população é representado por um vetor e uma matriz. O vetor representa quais das possíveis localizações de CDs irão estar efetivamente ativas. Assim, atribuindo o binário 1 à posição do vetor, um $\mathrm{CD}$ é habilitado no sistema, caso contrário o centro de distribuição não é habilitado. No exemplo da Figura 1, as localizações 1, 4, 29, 34, 38 e 44 recebem CD.

A segunda parte da representação da solução indica quais 
PV, serão atendidos por cada um dos CDs ativos. Para elaborar esta rotina, considera-se a distância de serviço $\mathrm{S}$ do ponto-de-venda aos CDs ativos. Caso exista mais de um CD candidato a atender certo $\mathrm{PV}$, a rotina deve escolher um $\mathrm{CD}$ apenas, verificando se a somatória dos volumes dos estoques alocados ao CD não ultrapassa sua capacidade. No exemplo da Figura 2, os 46 pontos-de-venda existentes (PVs) são alocados aos seis CDs ativos. $\mathrm{O}$ valor 1 indica a qual $\mathrm{CD}$ o PV foi alocado. Alguns PVs, como por exemplo 41, 42 e 43 , não foram alocados a nenhum $\mathrm{CD}$, ou porque estão fora da área de cobertura dos CDs, ou porque não há capacidade suficiente no CD para atendê-los.

A partir da criação da população inicial, a rotina de algoritmo genético seleciona dois indivíduos desta população, sendo um com o melhor fitness da população e o outro aleatoriamente ( $\mathrm{P} 1$ e $\mathrm{P} 2)$. A seguir, executa-se o crossover, criando-se dois indivíduos filhos (F1 e F2). Existe ainda uma probabilidade de ocorrência de operação de mutação sobre os filhos criados. Caso algum dos filhos possua fitness superior a algum dos indivíduos da população, é incluído na população. A seqüência de operações acima é realizada por um determinado número de gerações e a melhor solução obtida é apresentada.
A operação de crossover se dá por meio da escolha aleatória de dois pontos, nos vetores pais P1 e P2. Uma cadeia de string do vetor P1 é copiada para o vetor P2, gerando o filho F2. O mesmo processo ocorre para gerar o filho F1. Assim, uma cadeia de string é copiada do vetor P2 para o vetor P1 gerando o filho F1. Um exemplo de crossover é mostrado na Figura 3.

Com relação à matriz de alocação de PVs aos $\mathrm{CDs}$, o crossover se dá do seguinte modo: para cada PV, considerase quais os CDs ativos do filho e alocam-se os PVs ao CD do P1 ou do P2, mediante sorteio.

A mutação da matriz de CDs ativos ocorre com o sorteio aleatório de um bit, mudando seu valor, respeitando-se a restrição do número mínimo e máximo de CDs ativos. A mutação da matriz de alocação de PVs aos CDs se dá de forma análoga, ou seja, sorteia-se um PV e altera-se novamente mediante sorteio para qual novo CD este PV será alocado, desde que seja respeitada a distância de cobertura e a capacidade do CD.

O cálculo do fitness de um indivíduo se dá pelo total de vendas de todos os pontos, subtraído dos custos fixos de instalação dos CDs ativos e subtraídos dos custos dos estoques de segurança descentralizados e centralizados.

Figura 1: Exemplo de vetor soluc̣ão para localização de CD.

\begin{tabular}{|l|c|c|c|c|c|c|c|c|c|c|}
\hline \multicolumn{1}{|c|}{ VETOR SOLUÇOA } & $\mathbf{1 6}$ & $\mathbf{1 8}$ & $\mathbf{2 9}$ & $\mathbf{3 3}$ & $\mathbf{3 4}$ & $\mathbf{3 8}$ & $\mathbf{4 4}$ \\
\hline Possíveis CD's & $\mathbf{1}$ & $\mathbf{4}$ & $\mathbf{1 4}$ & $\mathbf{1 6}$ & $\mathbf{1 8}$ & 1 & 1 & 1 \\
\hline CD's Habilitados & 1 & 1 & 0 & 0 & 0 & 1 & 0 & 1 & 1 & 1 \\
\hline
\end{tabular}

Figura 2: Exemplo de vetor solução para alocação de pontos-de-vendas aos CDs.

\begin{tabular}{|c|c|c|c|c|c|c|c|c|c|c|c|c|c|c|c|c|c|c|c|}
\hline & \multicolumn{19}{|c|}{ PONTOS-DE-VENDA } \\
\hline CDs & 1 & 2 & 3 & 4 & 5 & 6 & 7 & 8 & 9 & 37 & 38 & 39 & 40 & 41 & 42 & 43 & 44 & 45 & 46 \\
\hline 1 & 0 & 0 & 1 & 0 & 0 & 0 & 0 & 0 & 0 & 0 & 0 & 0 & 0 & 0 & 0 & 0 & 0 & 0 & 1 \\
\hline 4 & 0 & 0 & 0 & 1 & 1 & 0 & 1 & 0 & 0 & 1 & 0 & 1 & 0 & 0 & 0 & 0 & 0 & 0 & 0 \\
\hline 14 & 0 & 0 & 0 & 0 & 0 & 0 & 0 & 0 & 0 & 0 & 0 & 0 & 0 & 0 & 0 & 0 & 0 & 0 & 0 \\
\hline 16 & 0 & 0 & 0 & 0 & 0 & 0 & 0 & 0 & 0 & 0 & 0 & 0 & 0 & 0 & 0 & 0 & 0 & 0 & 0 \\
\hline 18 & 0 & 0 & 0 & 0 & 0 & 0 & 0 & 0 & 0 & 0 & 0 & 0 & 0 & 0 & 0 & 0 & 0 & 0 & 0 \\
\hline 29 & 0 & 0 & 0 & 0 & 0 & 0 & 0 & 0 & 0 & 0 & 0 & 0 & 1 & 0 & 0 & 0 & 0 & 0 & 0 \\
\hline 33 & 0 & 0 & 0 & 0 & 0 & 0 & 0 & 0 & 0 & 0 & 0 & 0 & 0 & 0 & 0 & 0 & 0 & 0 & 0 \\
\hline 34 & 1 & 0 & 0 & 0 & 0 & 0 & 0 & 0 & 1 & 0 & 0 & 0 & 0 & 0 & 0 & 0 & 0 & 0 & 0 \\
\hline 38 & 0 & 0 & 0 & 0 & 0 & 0 & 0 & 0 & 0 & 0 & 1 & 0 & 0 & 0 & 0 & 0 & 0 & 1 & 0 \\
\hline 44 & 0 & 1 & 0 & 0 & 0 & 1 & 0 & 1 & 0 & 0 & 0 & 0 & 0 & 0 & 0 & 0 & 1 & 0 & 0 \\
\hline
\end{tabular}




\section{Parâmetros do algoritmo}

Os parâmetros do modelo matemático a serem fornecidos são: a distância máxima ou raio de cobertura de cada centro de distribuição, o número de iterações desejadas, o número mínimo e o número máximo de centros de distribuição. Já os parâmetros relacionados especificamente à execução do algoritmo genético são: a quantidade de elementos que a população deve conter e a probabilidade de mutação.

Os pontos-de-venda candidatos a terem a função de $\mathrm{PV}$ e CD foram os de número $1,4,14,16,18,29,33,34,38$ e 44 . Os dados coletados para a execução do projeto foram:

- O lucro bruto estimado de cada uma das farmácias que compõem o modelo no período de 1o de janeiro de 2004 a 31 de dezembro de 2004. O somatório foi estimado em $\mathrm{R} \$ 6.231 .185,00$. O valor foi calculado multiplicando as quantidades de itens comercializadas no ano de 2004 de cada linha de produto comercializada (genérico, ético, similar e perfumaria) em cada ponto-de-venda, pelos percentuais de lucratividade destas linhas. A soma do lucro dos pontos de 1 a 46 resulta no valor do lucro anual.

- Os custos fixos anuais estimados de cada um dos 10 pontos-de-vendas candidatos a atuarem como centro de distribuição dentro de sua área de cobertura.

- As coordenadas geográficas em UTM (Universal Transversa de Mer-

\section{APLICAC̣ÃO DO ALGORITMO EM UM PROBLEMA REAL}

O modelo implementado foi aplicado no estudo logístico de uma rede de farmácias localizada em uma capital de estado. A rede estudada é composta por cerca de 90 pontosde-venda. Decidiu-se então trabalhar com o total de pontos responsáveis por $80 \%$ do faturamento da rede, chegando-se assim a 46 pontos.

Dentre os 46 pontos-de-venda selecionados, foram escolhidos 10 pontos-de-venda candidatos a serem também localização de CDs. Esses pontos foram escolhidos por possuírem localização estratégica, acesso facilitado e área física disponível para o armazenamento de medicamentos. cator) de cada ponto-de-venda e candidato a CD considerado no modelo, utilizando-se um GPS.

- Os custos dos estoques de segurança atuais. O nível de estoque de segurança foi estimando como uma porcentagem do estoque médio. $\mathrm{O}$ valor do estoque de segurança descentralizado de todos os pontos foi calculado em $\mathrm{R} \$ 1.414 .632,75$.

$\mathrm{Na}$ execução do modelo foram utilizados os seguintes parâmetros:

- A distância máxima do raio de cobertura de cada centro de distribuição foi de $5 \mathrm{~km}$.

- O número de iterações em cada execução foi de 150 .

- O número mínimo de CDs igual a 1 , e o número máximo

Figura 3: Criando vetores filhos F1 e F2 via crossover.

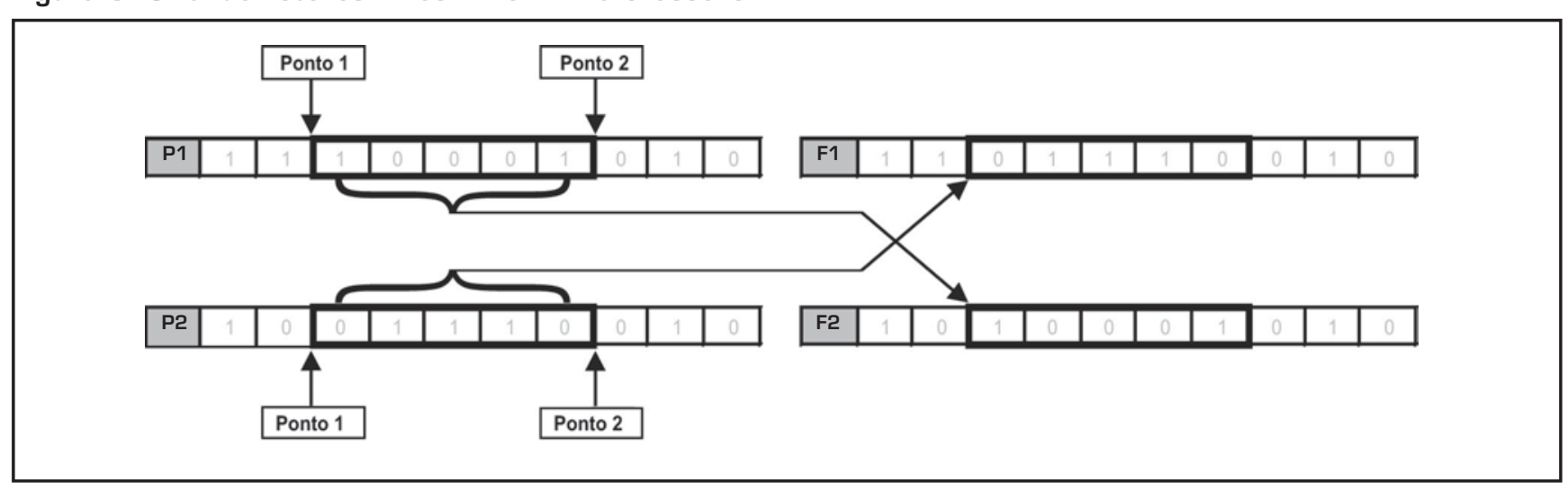


de CDs igual a 10.

- A probabilidade de mutação adotada foi de 3\%.

- O tamanho da população igual a 8 indivíduos.

\section{Resultados obtidos}

O software foi executado dez vezes seqüencialmente. O melhor resultado foi obtido na sétima tentativa, na iteração de número 72. A execução durou cerca de 5,657 segundos em um Pentium 4, com 256K de memória RAM.

Dentre os 10 pontos candidatos a atuarem como centro de distribuição, o software habilitou os pontos 4, 14, 16, 29, 38 e 44. Com os 6 centros de distribuição habilitados, tem-se a cobertura de 42 pontos-de-venda, com apenas 4 pontos de vendas não atendidos. A Figura 4 mostra a posição dos centros de distribuição ativos e dos pontos-de-venda. O mesmo resultado pode ser visto na Tabela 1 .

Os pontos 3, 17, 34 e 43, que são pontos-de-vendas fora da área de cobertura de centros de distribuição, tiveram o valor dos seus estoques de segurança adicionado ao custo total de estoque de segurança centralizado, pois serão pontos-de-venda que permanecerão vendendo com estoque próprio, como anteriormente. O resultado da soma dos estoques de segurança desses pontos-de-vendas foi de $\mathrm{R} \$ 120.742$, 45. A Figura 4 mostra a distribuição geográfica dos CDs.
Com os resultados obtidos após a centralização dos estoques de segurança e adicionados os estoques de segurança dos pontos-de-venda que não foram cobertos pelo sistema proposto de distribuição, obteve-se o custo total de estoque de segurança do sistema proposto. Este valor foi de R\$ 594. 609, 93, de acordo com a Tabela 2.

A tabela 3 mostra o resultado final do modelo. $\mathrm{Na}$ estratégia descentralizada atual, o lucro bruto dos 46 pontos-de-venda no ano de 2004 foi de R $\$ 6.231 .185,00$ e os custo de estoques de segurança descentralizados somaram R\$1.414. 632, 75, o que perfaz um resultado líquido de R\$4.816.552, 25.

Já na estratégia de criação de CDs e centralização dos estoques, a soma dos estoques de segurança dos pontos centralizados e dos não cobertos totaliza $\mathrm{R} \$ 594.609,93$, ou seja, uma economia em estoque de segurança de $\mathrm{R} \$ 820.022,82$, contra um investimento de R \$ 470.300,00 em instalação de CDs, resultando em uma economia de R $\$ 349.722,82$ se adotada a estratégia de centralização dos estoques proposta pelo software.

\section{CONCLUSÕES}

A elaboração de uma estratégia eficiente de distribuição envolve os passos de determinação do número de depósitos e sua localização e a estratégia de alocação de estoques dos

Tabela 1: CDs ativos e PVs atendidos.

\begin{tabular}{|c|c|c|c|c|c|c|c|c|c|c|c|c|c|c|c|c|}
\hline CDS ATIVOS & PVS POR GD & \multicolumn{10}{|c|}{ COBERTURA DE PONTOS-DE-VENDAS } \\
\hline $\mathbf{4}$ & $\mathbf{1 5}$ & 4 & 2 & 5 & 7 & 10 & 11 & 12 & 22 & 24 & 25 & 26 & 36 & 37 & 39 & 41 \\
\hline $\mathbf{1 4}$ & $\mathbf{3}$ & 14 & 20 & 21 & & & & & & & & & & & & \\
\hline $\mathbf{1 6}$ & $\mathbf{6}$ & 16 & 19 & 23 & 28 & 32 & 40 & & & & & & & & & \\
\hline $\mathbf{2 9}$ & $\mathbf{4}$ & 29 & 9 & 33 & 42 & & & & & & & & & & & \\
\hline $\mathbf{3 8}$ & $\mathbf{6}$ & 38 & 1 & 8 & 13 & 15 & 45 & & & & & & & & & \\
\hline $\mathbf{4 4}$ & $\mathbf{8}$ & 44 & 6 & 18 & 27 & 30 & 31 & 35 & 46 & & & & & & & \\
\hline
\end{tabular}

Tabela 2: Custo total do estoque de segurança do sistema proposto.

\begin{tabular}{|l|l|}
\multicolumn{1}{|c|}{ TOTAL DE ESTOQUES DA (AFDGO) } & CUSTO - RS \\
\hline Estoques centralizados & $473.867,47$ \\
\hline Estoques de pontos não atendidos & $120.742,45$ \\
\hline Total & $\mathbf{5 9 4 . 6 0 9 , 9 2}$ \\
\hline
\end{tabular}


produtos em cada um dos depósitos. Devido à complexidade envolvida em cada um desses passos, essas decisões têm sido tomadas de forma hierárquica e seqüencial, o que tem levado a obtenção de soluções sub-ótimas para o problema como um todo.

Este trabalho propõe e implementa um modelo conjunto para a solução dos problemas de localização de depósitos e alocação de estoques, unindo os conceitos de modelagem de problemas de máxima cobertura com a equação de Maister para a estimativa da redução dos estoques de segurança decorrentes da centralização dos mesmos.

Devido à complexidade combinatória envolvida no pro-

Figura 4: Configuração geográfica obtida por meio da execução do software.

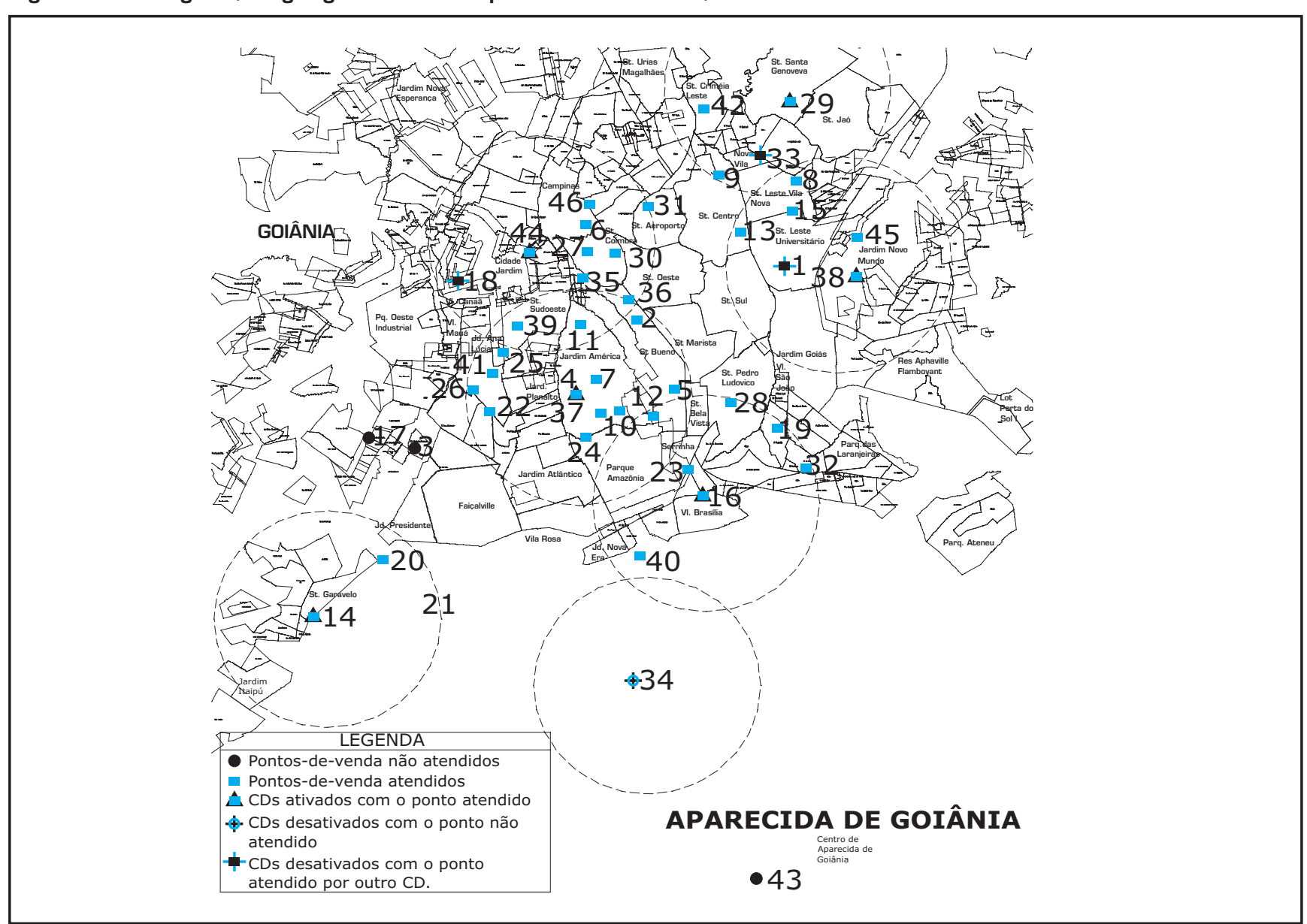

Tabela 3: Análise comparativa dos custos das duas estratégias.

\begin{tabular}{|l|c|c|}
\hline \multicolumn{2}{|c|}{ ESTRATÉGIA DE DISTRIBUIÇÃO ATUAL X PROPOSTA } \\
\hline Lucro anual & ATUAL (2004) & PROPOSTA \\
\hline Estoque de segurança & $R \$ 6.231 .185,00$ & $\mathrm{R} \$ 6.231 .185,00$ \\
\hline Custo fixo de CDs & $\mathrm{R} \$ 1.414 .632,75$ & $\mathrm{R} \$ 594.609,93$ \\
\hline Resultado & $\mathrm{R} \$ 0,00$ & $\mathrm{R} \$ 470.300,00$ \\
\hline
\end{tabular}


blema, a implementação se deu por meio de abordagem heurística, baseada em algoritmos genéticos. Estudos futuros deverão ser realizados de modo a se avaliar o grau de defasagem entre a qualidade da solução do modelo heurístico aqui proposto e a solução ótima para o problema. No estudo em questão, o modelo foi parametrizado para executar durante 150 gerações, o que levou cerca de 5 segundos e geralmente as melhores soluções não foram obtidas nas últimas iterações do modelo. Assim sendo, parece não haver problemas relacionados a tempo de execução para que sejam tratados problemas de porte bastante maior.

Com relação à parametrização do modelo, é importante ressaltar ainda que, segundo a literatura, em alguns casos a estimativa de redução dos estoques de segurança obtida pela lei da raiz quadrada pode ser bastante otimista. Assim, a elaboração de uma metodologia para a estimativa de redução dos estoques de segurança centralizados de forma mais precisa é recomendável. A mesma observação é válida para a estimativa dos custos fixos dos CDs.

O modelo heurístico proposto foi aplicado no estudo de uma rede de farmácias. Considerando-se os custos de estoque de segurança centralizados e descentralizados e os custos fixos de localização, o modelo aponta para o caso estudado uma economia de 349 mil reais. Estuda-se a implantação do modelo proposto por eta-

\title{
modelo heurístico proposto foi aplicado no estudo de uma rede de farmácias. Considerando-se os custos de estoque de segurança centralizados e descentralizados e os custos fixos de localização, o modelo aponta para o caso estudado uma economia de 349 mil reais.
}

\begin{abstract}
pas, ou seja, a implantação da centralização dos estoques, criando-se inicialmente um $\mathrm{CD}$ com um número intermediário de PVs atendidos (provavelmente o CD 16 ou o CD 38) de modo a refinar a parametrização do modelo. Com este CD piloto, será possível avaliar a real proporção de redução dos estoques de segurança, os reais custos de implantação dos CDs e eventuais impactos no atendimento a clientes, que podem vir a reduzir o faturamento.
\end{abstract}

\section{Artigo recebido em 21/06/2006 \\ Aprovado para publicação em 10/11/2006}

\section{- Referências Bibliográficas}

AVERBAKH, L. \& BERMAN, O. Routing and location-routing p-delivery men problems on a path. Transportation Sciences, v. 28, 1994.

BALLOU, R. H. Estimating and auditing aggregate inventory levels at multiple stocking points. Journal of Operations Management, v. 1, p. 143-153, 1981.

BALLOU, R. H. Logística empresarial: transportes, administração de materiais e distribuição física. 1. ed. São Paulo: Atlas, 1993.

BENVENISTES, R. A note on covering problem. Operations Research Society, p. 261-265, 1982.
BERMAN, O.; DRESNER, Z. and WESOLOWSKY, G. O. Minimum covering criterion for obnoxious facility location on network. Networks, v. 28, p. 1-5, 1996.

BOWERSOX, D. J \& CLOSS, D. J. Logística empresarial: o processo de integração da cadeia de suprimentos. 1. ed. São Paulo: Atlas, 2001.

BOWERSOX, D. J; CLOSS, D. J.; COOPER, M. B. Gestão logística da cadeia de suprimentos. tradução Camila Teixeira Nakagawa, Gabriela Teixeira Nakagawa, Porto Alegre: Bookman, 2006
BRYMAN, A. Research methods and organization studies. London: Routledge (Taylor\& Francis Group), 1989.

CHIEN, T. W. Heuristic procedures for practical sized uncapacited locationcapacited routing problems. Decision Sciences, v. 24, 1993.

CHRISTOPHER, M. Logística e gerenciamento da cadeia de suprimentos: estratégia para redução de custos e melhoria dos serviços. São Paulo: Pioneira, 1999.

CHURCH, R.L.; EATON, D. J. A bicriteriun maximal covering location formulation with considers the satisfaction of uncovered demand. Decisions Sciences, v. 22, p. 38-52, 1987.
CHURCH, R. L.; GARFUNKEL, R. S. Location an obnoxious facility on a network. Transportation Sciences, v. 12, n. 2, p. 107-118, 1978.

CHURCH, R.L.; REVELLE, C. The maximal covering location problem. Journal of Regional Science; v. 32, p. 101-118, 1974

DASKIN, M. S.; STERN, E. H. A hierarchical objective set covering model for emergency medical service vehicle deployment. Transportation Science; v. 15 , p. $137-152,1981$ 


\section{Referências Bibliográficas}

EPPEN, G. D. Effects of centralization on expected costs in a multi-location newsboy problem. Management Science, v. 25 , n. 5 , p. $498-501,1979$.

FITZSIMMONS, J. A.; FITZSIMMONS, M. J. Administração de serviços: operações, estratégia e tecnologia de informação. 2. ed. Porto Alegre: Bookman, 2000.

GOLDBARG, M. C. 0 problema de alocação ótima de radares de vigilância: um estudo por técnicas de cobertura. Dissertação de Mestrado, Instituto Militar de Engenharia, Rio de Janeiro, 1987.

GOUVEIA, L. M. B. Logística e gestão da distribuição: depósito e política de localizaç̃̃o. Porto, 1995.

JENKINS, C. H. Moderns warehouse management. New York: McGraw-Hill, 1968.
LAPORTE, G.; NORBERT, Y.; TAILLEFER, S. Solving a family of multi-depot vehicle routing and location-routing problems. Transportation Sciences, v. 22, 1998.

MAHMOUD, M. M. Optimal inventory consolidation schemes: a portfolio effect analysis. Journal of Business Logistics, $\mathrm{v}$. 13, n 1, p. 193-214, 1992

MAISTER, D. H. Centralization of inventories and the square root law. International Journal of Physical Distribution and Materials Management, n. 3, p. 124134, 1976.

MOREIRA, D. Administração da produção e operações. São Paulo: Pioneira, 1996.

MOTTA, I de S. Manual de administração da produção. Rio de Janeiro: Fundação Getúlio Vargas, 1990.
SAMBOLA, M. A. Models and algorithms for location-routing and related problems. Tese de Doutorado, Universidade Politécnica da Catalunha, Espanha, 2003.

SILVA, E. L.; MENEZES, E. M. Metodologia da pesquisa e elaboração de dissertação. 4. ed., Universidade Federal de Santa Catarina, Florianópolis, 138 p., 2005.

SILVEIRA, C. A. da. Centralização de estoques de materiais para as refinarias da Petrobrás situadas no estado de São Paulo. Dissertação de Mestrado. Programa de Pós Graduação em Engenharia de Produção, Universidade Federal de Santa Catarina, Florianópolis, 77 p., 2002.

SIMCHI-LEVI, D.; KAMINSKY, P.; SIMCHILEVI, E. Designing and managing the supply chain: concepts, strategies and case studies. Boston: McGraw-Hill, 2000.

SLACK, N; CHAMBERS, S.; HARLAND, C.; HARRISON, A.; JOHNSTON, R.
Administração da produção. São Paulo: Atlas, 1999.

SMYKAY, E. W. Physical distribution management. New York: Macmillan Publishing Co, 1973.

TALLON, W. J. The impact of inventory centralization on aggregate safety stock: the variable supply lead time case. Journal of Business Logistics, 1993.

VALE, C. E. Implantação de indústrias. Rio de Janeiro: Livros técnicos e científicos, 1975 .

WANKE, P.; FIGUEREDO, K. Logística empresarial: a perspectiva brasileira. São Paulo: Atlas, 2000.

WHITE, J.; CASE, K. On covering problems and the central facilities: a multicriteria approach. Decision Sciences, v. 4 , p. $712-714,1980$

\section{- Sobre os autores}

\section{Ricardo Ferrari Pacheco}

Universidade Católica de Goiás

Departamento de Engenharia

End.: Av. Universitária, 1069 - Área 3 - CP 86 - CEP 74.605-010

Tel.: (62) 8408-9296

E-mail: rpacheco@ucg.br

\section{Luciano Zorzett Cirqueira}

Lord Meat Ind. Com. Importação e Exportação Ltda.

Gerente de Projetos e Manutenção

End.: Go 230, Km 3,5, s/n - Zona Rural - Goianésia - GO

Tel.: (62) 3389-9700

E-mail: 1.cirqueira@1ordmeat.com.br 